\title{
Randomized clinical trial of sacral nerve stimulation for refractory constipation
}

\author{
F. Zerbib ${ }^{1}$, L. Siproudhis ${ }^{2}$, P.-A. Lehur ${ }^{3}$, C. Germain ${ }^{4}$, F. Mion ${ }^{5}$, A.-M. Leroi ${ }^{6}$, B. Coffin ${ }^{7}$, A. Le \\ Sidaner $^{8}$, V. Vitton ${ }^{9}$, C. Bouyssou-Cellier ${ }^{1}$ and G. Chene ${ }^{2}$, on behalf of the CONSTIMOD study \\ investigators
}

${ }^{1}$ Department of Gastroenterology, Bordeaux University Hospital, and Université de Bordeaux and Institut National de la Santé et de la Recherche Médicale (INSERM) CIC 1401, ${ }^{2}$ Department of Gastroenterology, Rennes University Hospital, and Université de Rennes, Rennes, ${ }^{3}$ Institut des Maladies de 1'Appareil Digestif, Nantes University Hospital, Nantes, ${ }^{4}$ Clinical Epidemiology Unit, and Bordeaux University Hospital and INSERM CIC 1401-EC, Bordeaux, ${ }^{5}$ Department of Digestive Physiology, Lyon University Hospital, and Université Claude Bernard, Lyon, ${ }^{6}$ Department of Digestive and Urinary Physiology, Rouen University Hospital, and Université de Rouen, Rouen, ${ }^{7}$ Department of Gastroenterology, Louis Mourier University Hospital, Assistance Publique - Hôpitaux de Paris, Colombes, and Université Denis Diderot Paris 7, Paris, ${ }^{8}$ Department of Gastroenterology, Limoges University Hospital, Limoges, and ${ }^{9}$ Department of Gastroenterology, Hôpital Nord, Assistance Publique - Hôpitaux de Marseille, and Plateforme d'Interface Clinique, CRN2M, Unité Mixte de Recherche 7286, and Aix Marseille Université, Marseille, France

Correspondence to: Professor F. Zerbib, Department of Gastroenterology and Hepatology, Haut Lévêque Hospital, Centre Hospitalier Universitaire de Bordeaux, Avenue Magellan, 33600 Pessac, France (e-mail: frank.zerbib@chu-bordeaux.fr) 
Presented to United European Gastroenterology Week, Barcelona, Spain, October 2015, () and Journées Francophones d'Hépato-gastroentérologie et d'Oncologie Digestive 2016, Paris, France, March 2016; published in abstract form as United European Gastroenterology Journal 2015;

3(Suppl): A21 
Background: Open studies have reported favourable results of sacral nerve stimulation for the treatment of refractory constipation. Here, its efficacy was assessed in a double-blind crossover RCT.

Methods: Patients with at least two of the following criteria were included: fewer than three bowel movements per week; straining to evacuate on more than 25 per cent of attempts; or sensation of incomplete evacuation on more than 25 per cent of occasions. Response to therapy was defined as at least three bowel movements per week and/or more than 50 per cent improvement in symptoms. Responders to an initial 3-week peripheral nerve evaluation were offered permanent implantation of a pulse generator and were assigned randomly in a crossover design to two 8-week intervals of active or sham stimulation. At the end of the two trial periods, the patients received active stimulation until the final evaluation at 1 year.

Results: Thirty-six patients (34 women, mean age 45(14) years) underwent peripheral nerve evaluation. Twenty responded and received a permanent stimulator. A positive response was observed in 12 of 20 and 11 of 20 patients after active and sham stimulation periods respectively $(P=0.746)$. Pain related to the device occurred in five patients and wound infection or haematoma in three, leading to definitive removal of the pulse generator in two patients. At 1 year, 11 of the 20 patients with an implanted device continued to respond. Stimulation had no significant effect on colonic transit time.

Conclusion: These results do not support the recommendation of permanent implantation of a pulse generator in patients with refractory constipation who initially responded to temporary nerve stimulation. Registration number: NCT01629303 (http://www.clinicaltrials.gov).

\section{+A: Introduction}

Chronic constipation is a common disorder in Western countries with a prevalence ranging from 15 to 30 per cent in the general population, being especially high in women and after the age of 65 years ${ }^{1-3}$. Most people can be treated successively with laxatives and/or bulking agents, but a subgroup of patients develop severe symptoms that are refractory to conservative therapies including biofeedback and irrigation, and result in significant deterioration in quality of life $(\mathrm{QoL})^{4}$. Refractory constipation 
is a complex disorder related to slow transit and/or impaired evacuation, for which subtotal colectomy with ileorectal anastomosis can be proposed in highly selected patients ${ }^{5}$. However, colectomy has a variable outcome ${ }^{5}$, and a recent nationwide survey ${ }^{6}$ in the USA has shown that it is associated with significant morbidity and does not decrease resource use.

Sacral nerve stimulation (SNS), a validated therapy for faecal incontinence ${ }^{7,8}$, has been suggested for patients with refractory constipation. Uncontrolled studies have reported encouraging results, with $42-100$ per cent of patients showing improvement during the test phase of SNS $^{9}$ and up to 87 per cent with sustained improvement at 2 years with permanent stimulation ${ }^{10}$. However, more recent studies have reported limited efficacy of $\mathrm{SNS}^{11}$. Furthermore, an $\mathrm{RCT}^{12}$ was unable to demonstrate a benefit of subsensory or suprasensory stimulation over sham stimulation in a series of 59 patients with slow-transit constipation. As underlined in the most recent revision of the Cochrane review $^{13}$, high-quality randomized trials are still warranted ${ }^{13}$. This aim of this RCT was to assess the efficacy of SNS in patients with refractory constipation.

\section{+A: Methods}

CONSTIMOD (Efficacy of Sacral Nerve Modulation in Severe Refractory Constipation) was designed as a multicentre, randomized, double-blind, placebo-controlled, crossover study (Fig. 1). Eligible patients were recruited from eight French university hospitals by medical teams with experience of SNS.

Patients meeting the inclusion criteria underwent an initial 3-week peripheral nerve evaluation (PNE). If symptoms improved at a predefined level, implantation of a permanent pulse generator was offered. After a 2-week wash-out period, patients were randomized to the two-interval crossover to receive 8 weeks of subsensory active stimulation (on) and sham stimulation (off), in random order. A 2-week wash-out separated the two trial periods. At the end of the second period, all patients entered a second phase of the study and received active stimulation, until week 50 after randomization. The patients were asked to maintain an unchanged dose of oral laxatives during baseline, PNE and randomized crossover periods. They could use oral bisacodyl as rescue therapy. 
The randomization list was computer-generated by the study statistician (in blocks of 4 , no stratification) and the randomization process was centralized. The randomization of each patient was obtained from the secure website of the Clinical Epidemiology Unit, Bordeaux University Hospital. Investigators entered patients' diary data on the website which automatically checked for inclusion criteria and then generated the treatment arm for responders to PNE (on-off versus off-on).

The study protocol was approved by the Comité de Protection des Personnes Sud-Ouest et Outre Mer III, and the trial was performed in compliance with the Helsinki Declaration and Good Clinical Practices. Informed consent was obtained from each patient before inclusion in the study. A detailed information leaflet was provided to patients and they were blinded to the assigned period of stimulation and sham treatments. During the test (PNE) and 'on' periods, patients were asked if the stimulation was perceived to ensure they had subsensory stimulation. For each centre, two different investigators were involved, one to perform stimulation settings and the other, blinded to the type of stimulation, to collect diaries and clinical data. The investigators performed stimulation settings without giving any information to the patients. During the study, the patients could not change the stimulation parameters on their own as no remote control was provided.

\section{+B: Patients}

Adult patients were eligible if they had chronic constipation (for over 1 year) defined by at least two of the following criteria: two or fewer complete bowel movements per week; straining to evacuate at more than 25 per cent of attempts (for example, use of enema or digital extraction); or sensation of incomplete evacuation after defaecation on more than 25 per cent of occasions. Other inclusion criteria were: absence of symptomatic response to standard therapies (including osmotic agents, faecal emollients, prokinetics (prucalopride), laxatives, dietary modification and biofeedback) for at least 3 months; and provision of written informed consent.

Patients were not deemed eligible if they had: constipation secondary to anorectal malformation, previous colorectal surgery or significant pelvic floor anatomical abnormality (such as rectocele or rectal prolapse); constipation secondary to neurological disorders and/or opiates; previous colorectal surgery of any type; or a contraindication to SNS (pregnancy, anatomical limitations preventing 
placement of an electrode, skin disease at risk of infection, psychiatric disease, cardiac pacemaker, need for MRI).

\section{+B: Peripheral nerve evaluation - test period}

PNE was performed under local or general anaesthesia, according to centre preference. The electrode (Electrode 3093, Interstim ${ }^{\circledR}$; Medtronic, Minneapolis, Minnesota, USA) was inserted into the sacral S2, S3, and S4 foramen. The sacral nerve root that provided the best perineal motor response and the best ipsilateral big toe response was chosen. The lead was then connected to an external stimulator (Medtronic 3625, Interstim ${ }^{\circledR}$ ). A 3-week period of continuous low-amplitude stimulation (set at maximal subsensory threshold; $14 \mathrm{~Hz}, 210 \mathrm{~ms}$ ) was started and each patient completed a bowel diary to assess the outcome during test stimulation. According to centre protocol, prophylactic antibiotics were given before implantation of the electrode and stimulator,

\section{$+B$ : Permanent implantation of pulse generator}

Responders to PNE were eligible for permanent implantation. The clinical response was considered positive if the patient fulfilled at least one of the following criteria: increase in evacuation frequency from less than two, to three or more bowel movements per week; reduction of at least 50 per cent in the number of defaecation episodes with straining; or decrease of at least 50 per cent in defaecation episodes with a sense of incomplete evacuation. The presence of these criteria was checked and validated automatically on the website. Permanent implantation of the internal pulse generator (IPG) was undertaken if both the investigator and the patient confirmed the clinical benefit of the stimulation. Implantation was performed under general or local anaesthesia. The electrode was connected to a stimulator (Stimulator 3023, Interstim ${ }^{\circledR}$ ) via a tunnelled extension cable and implanted subcutaneously in the lumbar region, ipsilateral to the stimulation electrode. Sensory threshold was determined with the IPG and stimulation was set at maximal subsensory threshold during 'on' periods. Investigators were asked to check for adequate stimulation at each visit, and they were not allowed to change the stimulation settings before the end of the two periods of the crossover.

\section{+B: Outcome measures}


The primary outcome measure was the proportion of patients with a response during each treatment period (stimulation on and off). Response was measured over the last 3 weeks of each of the 8-week treatment periods.

Secondary outcome measures were: short- and long-term clinical and physiological factors associated with response to temporary and permanent SNS, percentage of patients with a response at 1 year, effects of SNS on patients' daily bowel diary items, Wexner score, effect on QoL, visual analogue scale (VAS) score rating bowel habit, anorectal manometry parameters and colonic transit time.

\section{+B: Assessments}

A 3-week daily stool diary was completed during baseline, PNE, at the end of each period of the crossover, and at the end of the study after 1 year. The diary recorded the following items: stool frequency, number of defaecations with straining, use of enemas or digital extraction, sensation of incomplete evacuation, time spent in the lavatory, abdominal pain (moderate or severe), bloating (moderate or severe) and the use of laxatives. At each visit, a VAS score was completed, asking patients to rate their bowel habit from 0 to 100 . Constipation severity was assessed using the Wexner score (Cleveland Clinic constipation score) ranging from 0 (no symptoms) to 30 (severe symptoms) $^{14}$. The effects on QoL were assessed by completion of the Gastrointestinal Quality of Life Index (GIQLI) questionnaire ${ }^{15}$ at baseline, at the end of each visit of the crossover period and at the end of follow-up. Anorectal manometry was undertaken and colonic transit time was calculated at baseline and at the end of follow-up. Anorectal manometry was performed with a single-use catheter to determine mean resting and squeeze pressure, rectal sensation to balloon distension with air, rectal sensory threshold, urge threshold and maximal tolerated rectal volume. Total colonic transit time was evaluated according to a previously validated technique ${ }^{16}$. The patient ingested ten radio-opaque markers per day for 6 days and underwent plain abdominal radiography day 7 to determine the number of retained markers. Colonic transit time was obtained by multiplying the number of markers by $2.4^{16}$. According to normal data obtained in healthy controls, slow colonic transit was defined by a value exceeding 90 h. The occurrence of adverse events was reported at each visit. Adverse event documentation included 
date reported, date of onset, description, severity, action taken, and assessment by the investigator whether the adverse event was related or possibly related to the device or study procedures. These events were classified as serious according to conventional definitions.

\section{+B: Statistical analysis}

For the primary objective, it was hypothesized that 30 per cent of patients would be responders with sham stimulation and active SNS would increase this proportion by a further 50 per cent ( 80 per cent responders). A total of 20 patients would give a power of at least 80 per cent, with a two-sided type I error of 5 per cent ( $\chi^{2}$ test). Based on previous studies ${ }^{9}$, reporting that $40-75$ per cent of patients (median 50 per cent) were responders after an initial period of stimulation and could receive a permanent implant, it was decided to include 40 patients in total to ensure that 20 patients would enter the randomized treatment phase.

The primary analysis was performed on an intention-to-treat basis with missing data considered as failure. A per-protocol analysis was also performed.

Continuous data are expressed as mean(s.d.) if distributed normally and median (range) otherwise, with analysis by Student's $t$ test and Wilcoxon test respectively. The $\chi^{2}$ test, or Fisher's exact test for expected frequencies below 5, was used to compare proportions between groups for categorical variables Two-sided 95 per cent confidence intervals (c.i.) of the difference in proportions were estimated by means of an asymptotic method.

Within-subject differences (PNE and 1-year visit versus baseline) were compared for quantitative variables by a Student's $t$ test or Wilcoxon test for matched continuous data, and by MacNemar $\chi^{2}$ test or exact test for qualitative variables.

Analysis of the crossover design for primary and secondary endpoints used mixed logistics regression models including type of stimulation and administration period as fixed effects and patient as random effect. Considering the challenges to estimate a co-variance matrix of fixed effects in the context of a small sample size, the final model for the primary outcome did not involve random effects. All statistical analyses were conducted using $\mathrm{SAS}^{\circledR}$ software version 9.2 (SAS Institute, Cary, North Carolina, USA). 


\section{$+\mathrm{A}:$ Results}

Between July 2012 and November 2013, 43 patients were screened, and 36 were included in eight centres (34 women; mean(s.d.) age 45(14) (range 18-69) years) (Fig. 2). The mean duration of constipation was 16.1(14.8) years. Patients had a mean of 5.6(11.2) bowel movements per week; a high proportion complained of straining at defaecation (29 of 36) or a sensation of incomplete evacuation (33 of 36). The mean Wexner score was 20.4(3.7). Previous treatments for constipation had failed, including enemas and suppositories (36 patients), laxatives (35), biofeedback (25) and prucalopride (24). Physiological characteristics are summarized in Table 1. The mean colonic transit time was $113.5(32.9) \mathrm{h}$ and 28 of 36 patients had value exceeding $90 \mathrm{~h}$. Anismus refractory to biofeedback was present in 13 patients. No complication was reported during PNE. The median sensory threshold was 1.0 (range $0.01-10) \mathrm{V}$.

\section{+B: Response to peripheral nerve evaluation}

Twenty of the 36 patients had a response according to study criteria (19 women, mean age 44(15) (range 18-67) years) and were randomized (Table 1). As planned in the trial protocol, recruitment stopped. once 20 patients had been randomized. The electrodes were inserted in S3 in 17 patients and S4 in three. The median sensory threshold was $1.0(0.5-10) \mathrm{V}$. No predictor of response to PNE could be identified (data not shown). Virtually all items of the daily stool diary improved, as well as Wexner and VAS scores, in responders to PNE (Table 2).

\section{+B: Primary outcome measure}

During the crossover period, the intention-to-treat analysis showed that 12 of 20 and 11 of 20 patients responded during the on and off periods respectively (unadjusted difference 5 (95 per cent c.i. -26 to 36) per cent; adjusted $P=0.746$ ) (Fig. 3). In this analysis one patient with missing data on the sham stimulation and two patients with missing data on the active stimulation were considered as nonresponders. No difference was shown between the first 8 weeks versus the second 8 weeks $(P=$ 0.339). The interaction between type of stimulation and stimulation period was not significant $(P=$ 0.734); the results shown are those of the model without interaction. Similar results were seen in the per-protocol analysis (data not shown). 


\section{$+B$ : Secondary outcome measures}

During the crossover period, there was no statistically significant difference between on and off periods for any item of the daily stool diary, Wexner score, VAS score (Table 2) or QoL scores (Fig. 3). There was no factor associated with response to SNS during this interval.

At 1 year, 16 patients were available for the follow-up assessment; two had had the stimulator explanted owing to wound infection, one withdrew consent because of pain and lack of efficacy, and one was excluded because lack of compliance to the study protocol and was lost to follow-up. Eleven patients had a sustained clinical response according the trial definition, and 14 wished to continue SNS. Among the 36 patients initially tested, no clinical or physiological factor was associated with a sustained clinical response. Among the 20 patients who responded to PNE and randomized, factors associated with a sustained response were older age (mean 51 versus 32 years; $P=0.017$ ), more than two bowel movements per week ( 4 of 5 versus 2 of 11; $P=0.036$ ), presence of severe dyschezia ( 9 of 11 versus 1 of $5 ; P=0.036$ ), and a lower maximal tolerable volume at anorectal manometry at the baseline visit (mean 167.8(63.5) versus 258.0(87.6) $\mathrm{ml} ; P=0.045$ ). Compared with baseline, at 1 year SNS was associated with an improvement in all stool diary items, which was statistically significant for defaecation with sensation of incomplete evacuation, number of days with moderate to severe pain, and Wexner and VAS scores (Table 2).

At 1 year, SNS was associated with a significant improvement in QoL for symptoms $(P<$ 0.001), physical condition $(P=0.003)$ and emotions $(P=0.004)$ (Fig. 4).

SNS was not associated with any significant modification of anorectal manometry parameters after 1 year. Mean colonic transit times were 113.5(32.9) and 108.2(38.5) h at inclusion and at 1 year respectively (mean(s.d.) difference $-12.8(36.1) \mathrm{h} ; P=0.226$ ).

\section{+B: Adverse events}

Nine serious adverse events occurred in eight patients, related to pain, infection (2) or dysfunction of the device (4) (Table 3). A total of 25 adverse events related to, or possibly related to the device were reported in 11 patients. Three patients had wound infection or haematoma, treated with antibiotics in one and definitive removal of the device in the other two. Two patients had an electrode replaced before randomization ( 1 for wire displacement, 1 for pain at stimulator site). 


\section{+A: Discussion}

In patients who responded to PNE and were given an IPG, this randomized crossover double-blind study could not demonstrate any significant effect of active compared with sham stimulation. After 1 year, 11 of 12 patients with an implanted stimulator remained responders to the therapy. Stimulation had no significant effect on colonic transit time.

The results of this study are consistent with those of a recent randomized crossover trial reported by Dinning and colleagues ${ }^{12}$ in patients with slow-transit constipation without evidence of evacuatory dysfunction. Most patients in the present trial had an increased colonic transit time, but patients with rectal evacuation disorders were not excluded as the potential mechanisms of action of SNS remain unclear ${ }^{17-19}$. Therefore, the approach was pragmatic, based on symptoms of constipation, such as low number of stools and difficult evacuation, refractory to conservative therapy. In that respect, inclusion criteria were similar to those of most studies reported to date ${ }^{9}$. Criteria for response to stimulation were similar to those reported by Kamm and co-workers ${ }^{10}$ : increase in evacuation frequency to three or more bowel movements per week, or reduction by at least 50 per cent in the number of defaecation episodes with straining or with sensation of incomplete evacuation. Based on these criteria, 20 of 36 patients in the present study were considered as responders to PNE, consistent with previous reports ${ }^{9}$. In contrast to the randomized trial reported by Dinning and colleagues ${ }^{12}$, only responders to PNE were offered a permanent IPG and randomized. Although PNE may predict the response to SNS in patients with faecal incontinence ${ }^{20}$, the authors acknowledge that it was not clear whether a failed PNE was associated with failure of chronic SNS in the context of chronic constipation at the time the study was designed. However, only a subgroup of patients with constipation may benefit from SNS; in the absence of clear predictors of response, and taken the cost and morbidity of the technique, it was considered too expensive and unfair to implant a stimulator in patients who did not respond to PNE. The finding of Dinning et al. ${ }^{12}$ that failed PNE was a good indicator of failed chronic stimulation validates this decision a posteriori.

The most important finding of the present study is that active stimulation had no significant effect compared with sham stimulation in both intention-to-treat (response in 12 of 20 versus 11 of 20 patients respectively) and per-protocol analyses. This is consistent with the results reported by Dinning 
and co-workers ${ }^{12}$, who used both subsensory and suprasensory stimulations. To date, only one randomized crossover trial ${ }^{18}$ has reported a significant improvement during active short-term $(2$ weeks) temporary stimulation in a very specific subgroup of patients with rectal evacuation disorders associated with rectal hyposensitivity.

The present trial some limitations. The sample size was calculated based on an expected placebo effect of 30 per cent and the hypothesis of an additional 50 per cent success rate achieved by active stimulation. Although such a difference might seem optimistic, the authors' group of experienced gastroenterologists and surgeons assumed that SNS would have to reach such a clinically meaningful effect to balance the potential risks and cost of the device. Such a sample size had limited power to identify predictive characteristics of benefit, and it was not possible to isolate a subgroup of participants who could be offered the device even in the context of no efficacy overall. A potential carry-over effect may explain the absence of difference between the active and sham stimulation periods, but the statistical analysis ruled out an order effect during the crossover period. The clinical response was measured during the last 3 weeks of each period of stimulation, giving a 7-week washout interval before assessment of the sham periods during the crossover (Fig. 1). Of note, only 12 of 20 patients responded during the active stimulation period, even though all of them initially responded to PNE. This relatively rapid loss of efficacy may be interpreted as a placebo effect of the PNE test.

During the crossover period, several items of the daily stool diary improved with active stimulation (Table 1), but without statistical significance because of stimulation type-period interaction and/or lack of power, as the study was designed to demonstrate an effect on response rates only.

At 1 year, only 16 patients were available for evaluation, 11 with a sustained clinical response, representing about one-third of the patients initially tested. Variable sustained response rates to chronic SNS have been reported, ranging from 11 to 63 per cent of patients tested ${ }^{10,11,21,22}$. Among those available for evaluation at 1 year, several symptoms of constipation were improved significantly compared with baseline, as well as three dimensions of the QoL questionnaires. Like others, the authors could not identify any clinical or physiological factor associated with sustained response to SNS in patients initially tested, and it is therefore difficult to predict the best candidates for this 
treatment. However, among the 20 randomized patients, a sustained clinical response at 1 year was more frequent in older patients with more than two bowel movements per week, severe dyschezia and a low maximal tolerable volume at anorectal manometry. In contrast to two studies ${ }^{10,18}$ in which SNS decreased sensory thresholds to rectal balloon distension, no effect of SNS on anorectal manometry parameters at 1 year was demonstrated here. The same holds true for colonic transit time, whereas Kamm et al. ${ }^{10}$ reported an acceleration of whole-gut transit time at 6 months. Apart from differences in methods used in these studies, the reasons for these discrepancies are not clear.

A total of 25 adverse events possibly related to the device occurred in the trial. This high rate, which is comparable to rates reported by other groups ${ }^{9}$, occurred despite the appreciable experience of SNS in the groups involved in this multicentre study. Maeda and co-workers ${ }^{23}$ reported 58 adverse events in 38 patients, 19 requiring surgical intervention. Despite the use of prophylactic antibiotics, as recommended by a recent European consensus statement ${ }^{7}$, three infections occurred, one of which was treated medically and two led to removal of the device.

This randomized crossover study did not show an effect of active stimulation compared with absence of stimulation in patients with refractory constipation who responded to PNE. Given the cost of the device and the risk of adverse events, the results do not support the recommendation to use SNS in patients with refractory constipation.

\section{+ A : Collaborators}

The CONSTIMOD study investigators are: F. Zerbib, M. Simon, Q. Denost, P. Lepicard (Bordeaux); P.-A. Lehur, G. Meurette, V. Wyart, C. Kubis (Nantes); F. Mion, S. Roman, H. Damon, X. Barth (Lyon), A.-M. Leroi, V. Bridoux, G. Gourcerol (Rouen); B. Coffin, B. Castel, C. Gorbatchef (Colombes); Anne Le Sidaner, Muriel Mathonnet (Limoges); V. Vitton, N. Lesavre, P. Orsoni (Marseille); L. Siproudhis, C. Brochard, V. Desfourneaux (Rennes).

\section{+A: Acknowledgements}

The authors are grateful to C. Keywood for English proofing of the manuscript. This study was funded by a grant from the French Ministry of Health (PHRC 2011) and promoted by Bordeaux University Hospital (Délégation à la Recherche Clinique, Centre Hospitalier Universitaire de Bordeaux). Medtronic provided three IPGs and supported one investigator meeting in Paris. Medtronic was not 
involved in the study design, conduct of the study or data analysis. F.Z. reports personal fees from Medtronic during the conduct of the study, and personal fees from Menarini, Coloplast, Shire and Almirall outside the submitted work. A.-M.L. reports personal fees from Medtronic during the conduct of the study, and from Wellspect outside the submitted work; P.-A.L. reports grants from Direction Générale de l'Organisation Sanitaire, French Ministry of Health, during the conduct of the study, and personal fees from a consultancy agreement with Medtronic SA outside the submitted work. F.M. reports personal fees from Medtronic during the conduct of the study.

Disclosure: The authors declare no other conflict of interest. 


\section{$+\mathrm{A}:$ References}

1 Sonnenberg A, Koch TR. Epidemiology of constipation in the United States. Dis Colon Rectum 1989; 32: 1-8.

2 Pare P, Ferrazzi S, Thompson WG, Irvine EJ, Rance L. An epidemiological survey of constipation in Canada: definitions, rates, demographics, and predictors of health care seeking. Am J Gastroenterol 2001; 96: 3130-3137.

3 Stewart WF, Liberman JN, Sandler RS, Woods MS, Stemhagen A, Chee E et al. Epidemiology of constipation (EPOC) study in the United States: relation of clinical subtypes to sociodemographic features. Am J Gastroenterol 1999; 94: 3530-3540.

4 Belsey J, Greenfield S, Candy D, Geraint M. Systematic review: impact of constipation on quality of life in adults and children. Aliment Pharmacol Ther 2010; 31: 938-949.

5 Knowles CH. Defecation: colectomy for constipation - a time for renewed caution? Nat Rev Gastroenterol Hepatol 2015; 12: 675-676.

6 Dudekula A, Huftless S, Bielefeldt K. Colectomy for constipation: time trends and impact based on the US Nationwide Inpatient Sample, 1998-2011. Aliment Pharmacol Ther 2015; 42: 12811293.

7 Maeda Y, O’Connell PR, Lehur PA, Matzel KE, Laurberg S; European SNS Bowel Study

Group. Sacral nerve stimulation for faecal incontinence and constipation: a European consensus statement. Colorectal Dis 2015; 17: O74-87.

8 Leroi AM, Damon H, Faucheron JL, Lehur PA, Siproudhis L, Slim K et al. Sacral nerve stimulation in faecal incontinence: position statement based on a collective experience. Colorectal Dis 2009; 11: 572-583.

9 Thomas GP, Dudding TC, Rahbour G, Nicholls RJ, Vaizey CJ. Sacral nerve stimulation for constipation. Br J Surg 2013; 100: 174-181.

10 Kamm MA, Dudding TC, Melenhorst J, Jarrett M, Wang Z, Buntzen S et al. Sacral nerve stimulation for intractable constipation. Gut 2010; 59: 333-340. 
stimulation for chronic constipation. Neurogastroenterol Motil 2015; 27: 734-739.

12 Dinning PG, Hunt L, Patton V, Zhang T, Szczesniak M, Gebski V et al. Treatment efficacy of sacral nerve stimulation in slow transit constipation: a two-phase, double-blind randomized controlled crossover study. Am J Gastroenterol 2015; 110: 733-740.

13 <JCIT>Thaha MA, Abukar AA, Thin NN, Ramsanahie A, Knowles CH. Sacral nerve stimulation for faecal incontinence and constipation in adults. Cochrane Database Syst Rev 2015; (8)CD004464.

14 Agachan F, Chen T, Pfeifer J, Reissman P, Wexner SD. A constipation scoring system to simplify evaluation and management of constipated patients. Dis Colon Rectum 1996; 39: 681-685. 15 Eypasch E, Williams JI, Wood-Dauphinee S, Ure BM, Schmulling C, Neugebauer E et al. Gastrointestinal Quality of Life Index: development, validation and application of a new instrument. Br J Surg 1995; 82: 216-222.

16 Danquechin Dorval E, Barbieux JP, Picon L, Alison D, Codjovi P, Rouleau P. [Simplified measurement of colonic transit time by one radiography of the abdomen and a single type of marker. Normal values in 82 volunteers related to the sexes.] Gastroenterol Clin Biol 1994; 18: 141-144. 17 Dinning PG, Hunt LM, Arkwright JW, Patton V, Szczesniak MM, Wiklendt L et al. Pancolonic motor response to subsensory and suprasensory sacral nerve stimulation in patients with slow-transit constipation. Br J Surg 2012; 99: 1002-1010.

18 Knowles CH, Thin N, Gill K, Bhan C, Grimmer K, Lunniss PJ et al. Prospective randomized double-blind study of temporary sacral nerve stimulation in patients with rectal evacuatory dysfunction and rectal hyposensitivity. Ann Surg 2012; 255: 643-649.

19 Carrington EV, Evers J, Grossi U, Dinning PG, Scott SM, O’Connell PR et al. A systematic review of sacral nerve stimulation mechanisms in the treatment of fecal incontinence and constipation. Neurogastroenterol Motil 2014; 26: 1222-1237.

20. Dudding TC, Pares D, Vaizey CJ, Kamm MA. Predictive factors for successful sacral nerve stimulation in the treatment of faecal incontinence: a 10-year cohort analysis. Colorectal Dis 2008; 10: 249-256. 
21. Ratto C, Ganio E, Naldini G, Gins. Long-term results following sacral nerve stimulation for chronic constipation. Colorectal Dis 2015; 17: 320-328.

22 Govaert B, Maeda Y, Alberga J, Buntzen S, Laurberg S, Baeten CG. Medium-term outcome of sacral nerve modulation for constipation. Dis Colon Rectum 2012; 55: 26-31.

23 Maeda Y, Lundby L, Buntzen S, Laurberg S. Sacral nerve stimulation for constipation: suboptimal outcome and adverse events. Dis Colon Rectum 2010; 53: 995-999.

\section{Typesetter: please refer to marked-up figures}

Sacral nerve stimulation for refractory constipation

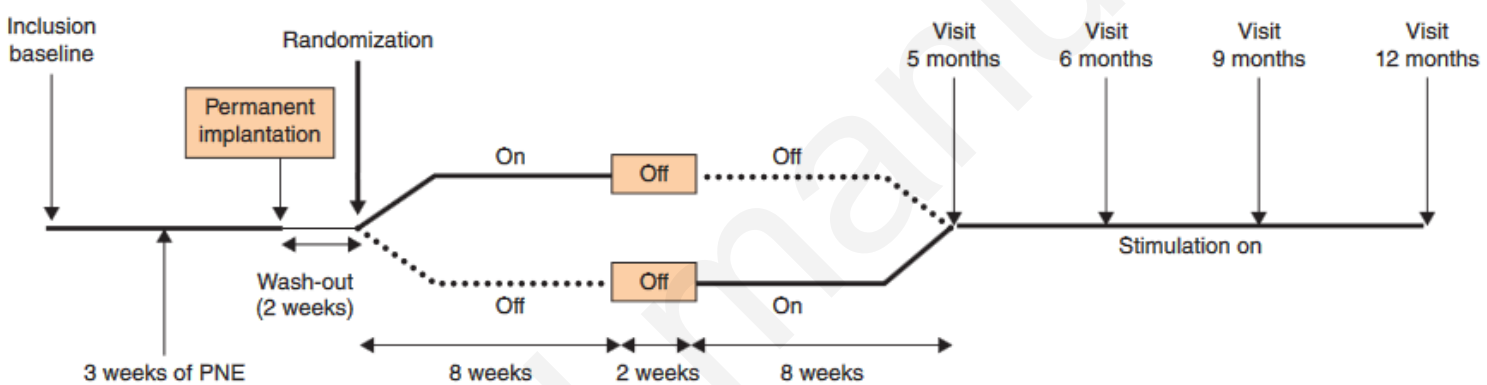

Fig. 1 Study design. Patients who responded to peripheral nerve evaluation (PNE) were offered permanent stimulator implantation and were randomised in a crossover design to have successive 8week periods of active (on) and sham (off) stimulation. After the crossover period, all patients were on active stimulation until the last visit at 1 year 


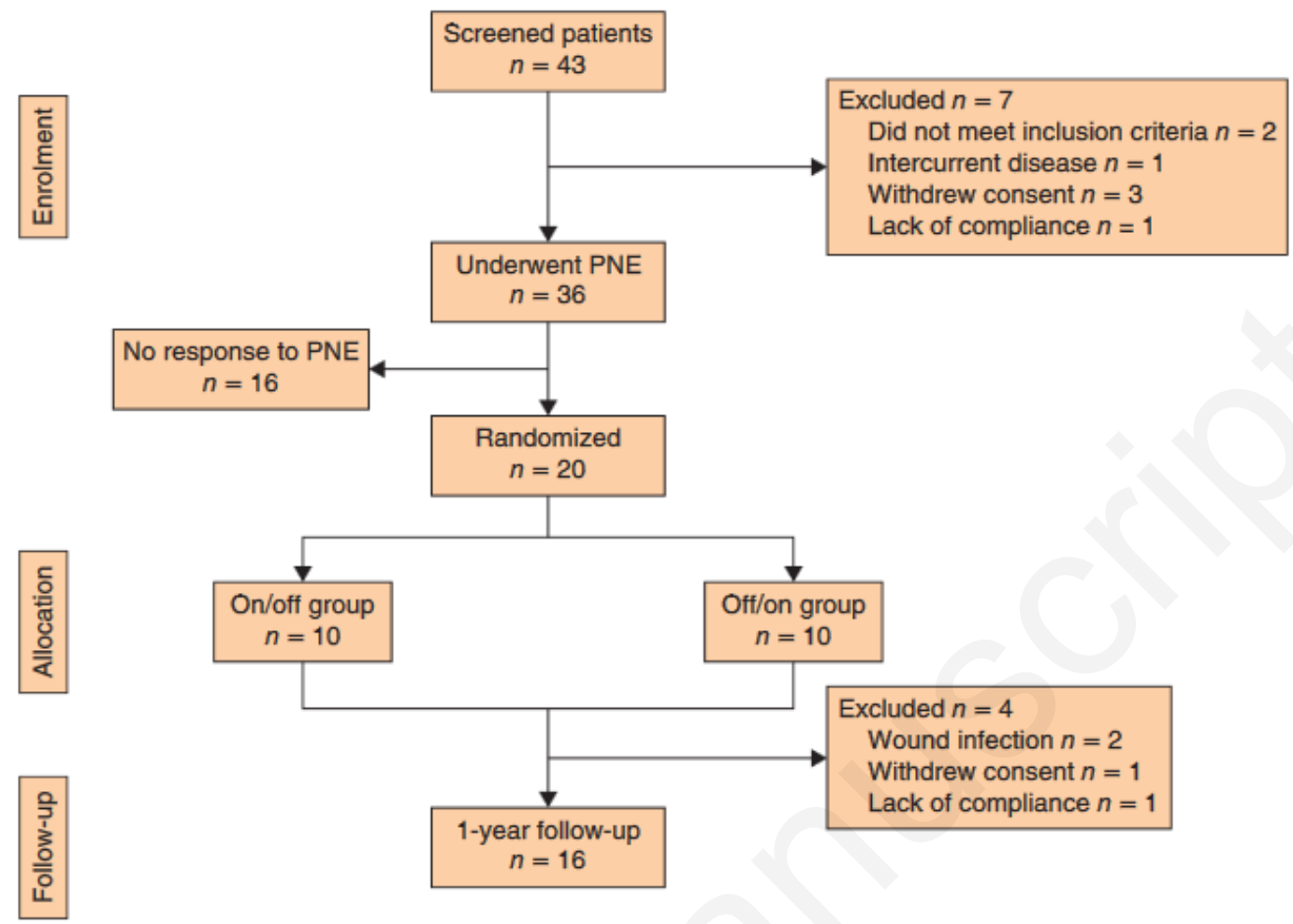

Fig. 2 Study flow chart. PNE, peripheral nerve evaluation

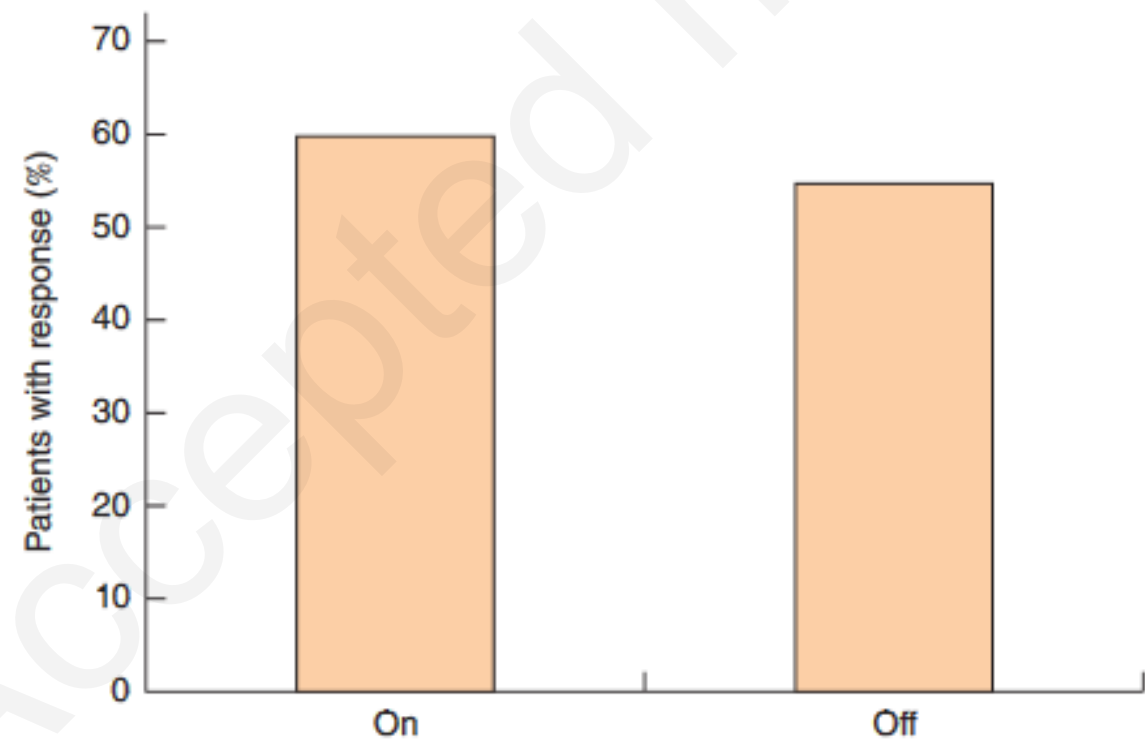

Fig. 3 Percentage of patients responding to sacral nerve stimulation during the crossover period (intention-to-treat analysis including 20 patients) 


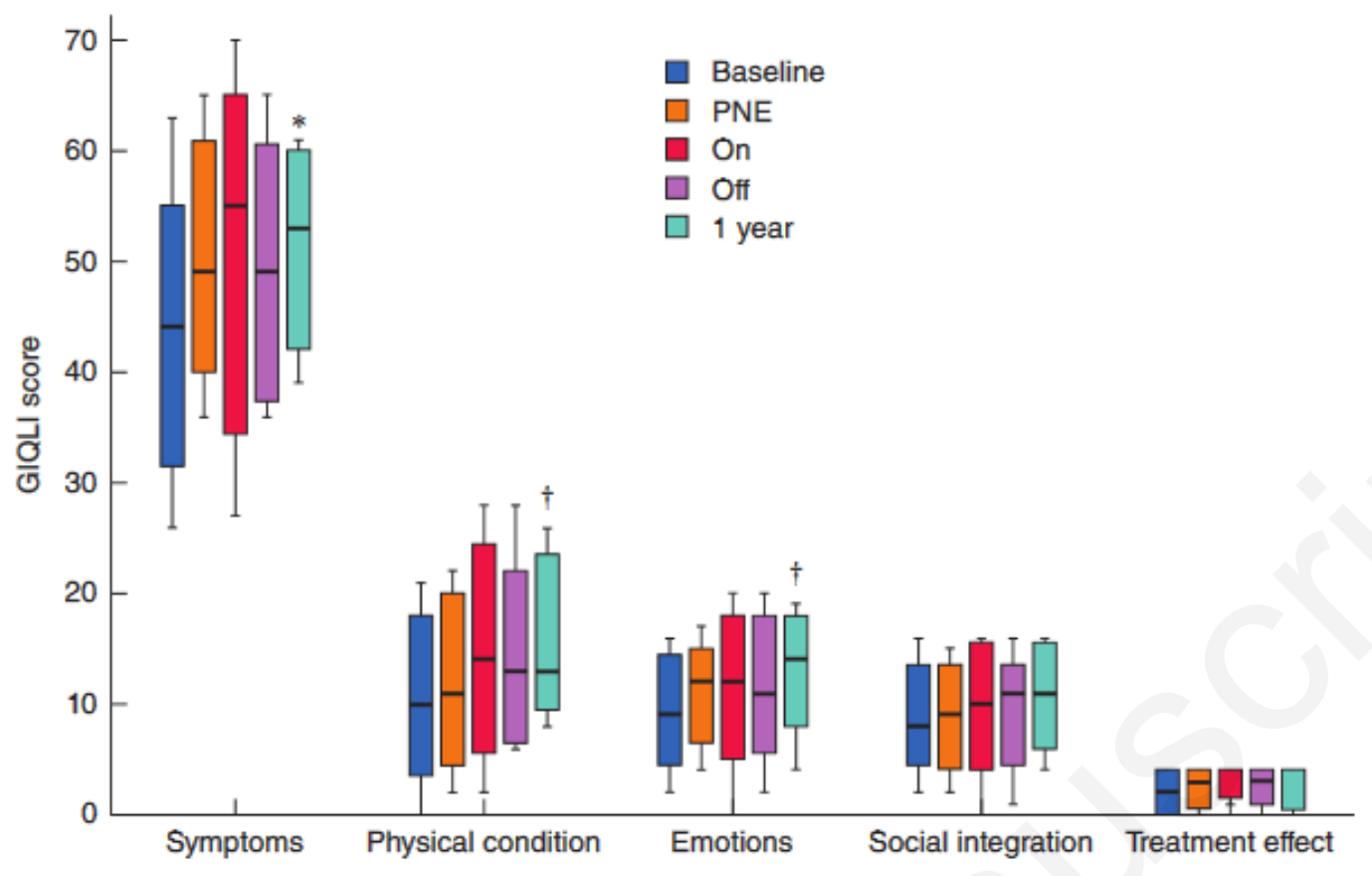

Fig. 4 Effects of sacral nerve stimulation on the five dimensions of the Gastrointestinal Quality of Life Index (GIQLI) score. PNE, peripheral nerve evaluation. Median (bold line), interquartile range (box) and range (error bars) are shown. $* P<0.001, \dagger P<0.010$ versus baseline (Wilcoxon paired test for comparisons versus baseline, and mixed logistic regression model for comparisons between on and off periods) 
Table 1 Baseline characteristics of 36 patients who underwent peripheral nerve evaluation, of whom 20 were randomized

\begin{tabular}{|c|c|c|}
\hline & $\begin{array}{l}\text { Patients included } \\
\qquad(n=36)\end{array}$ & $\begin{array}{l}\text { Patients randomized } \\
\qquad(n=20)\end{array}$ \\
\hline Age (years)* & $45(14)$ & $44(15)$ \\
\hline Sex ratio $(F: M)$ & $34: 2$ & $19: 1$ \\
\hline Colonic transit time $(\mathrm{h})^{*}$ & $113.5(32.9)$ & $115.5(29.8)$ \\
\hline Colonic transit time $>90 \mathrm{~h}$ & 28 & 16 \\
\hline Mean sensory threshold $(\mathrm{ml})^{*}$ & $46.7(50.9)$ & $55.8(63.3)$ \\
\hline Mean urge threshold $(\mathrm{ml})^{*}$ & $136.7(82.5)$ & 151.2(87.4) \\
\hline Mean maximal tolerable volume $(\mathrm{ml})^{*}$ & 209.7(86.8) & 206.4(83.4) \\
\hline Anismus & 13 & 7 \\
\hline
\end{tabular}

*Values are mean(s.d.) 
Table 2 Effects of sacral nerve stimulation on daily stool diary items, Wexner and bowel habit scores

\begin{tabular}{|c|c|c|c|c|c|}
\hline & $\begin{array}{l}\text { Baseline } \\
(n=20)\end{array}$ & $\begin{array}{c}\text { PNE } \\
(n=20)\end{array}$ & $\begin{array}{l}\text { On period } \\
(n=20)\end{array}$ & $\begin{array}{l}\text { Off period } \\
(n=20)\end{array}$ & $\begin{array}{l}12 \text { months } \\
(n=16)\end{array}$ \\
\hline Stool frequency & $11.0(9.2)$ & $20.9(13.8) \div$ & $22.6(25.5)$ & $16.5(19.8)$ & $17.4(16.7)$ \\
\hline $\begin{array}{l}\text { Use of enemas/suppositories, } \\
\text { digital extraction }(\%)^{*}\end{array}$ & $38.9(31.2)$ & $9.2(17.6) \S$ & $14.0(23.7)$ & $28.5(33.1)$ & $25.2(27.8)$ \\
\hline Defaecation with straining $(\%)^{*}$ & $52.8(39.8)$ & $28.2(34.4) \dagger$ & $28.1(39.3)$ & $28.5(42.1)$ & $43.1(45.2)$ \\
\hline $\begin{array}{l}\text { Defaecation with sensation of } \\
\text { incomplete evacuation }(\%)^{*}\end{array}$ & $70.9(36.5)$ & $34.0(31.7) \dagger$ & $50.9(44.5)$ & $57.2(41.5)$ & $54.3(39.6) \dagger$ \\
\hline Doses of bisacodyl & $23.2(44.0)$ & $9.8(19.5)$ & $11.7(20.7)$ & $22.5(41.1)$ & $14.3(23.3)$ \\
\hline Time spent in toilet (min) & $226.1(199.0)$ & $152.3(108.4) \dagger$ & $168.6(150.2)$ & $176.3(187.4)$ & $159.8(135.1)$ \\
\hline $\begin{array}{l}\text { Time experiencing moderate to } \\
\text { severe pain (days) }\end{array}$ & $17.4(5.5)$ & $10.0(7.5) \S$ & $13.1(8.4)$ & $15.8(7.3)$ & $12.6(7.6) \dagger$ \\
\hline $\begin{array}{l}\text { Time experiencing moderate to } \\
\text { severe bloating (days) }\end{array}$ & $19.7(2.3)$ & $12.2(7.3) \S$ & $14.9(7.5)$ & $17.2(5.7)$ & $16.7(6.9)$ \\
\hline Wexner score & $20.1(4.2)$ & $11.1(5.4) \S$ & $14.5(6.0)$ & $16.4(5.9)$ & $14.0(5.6) \dagger$ \\
\hline VAS score & 43.3(19.7) & $62.9(20.7)+$ & $53.8(29.2)$ & $44.3(30.2)$ & $64.3(26.2) \dagger$ \\
\hline
\end{tabular}

Values are mean(s.d.) and refer to 3-week intervals. *Percentage of defaecations. VAS, visual analogue scale from 0 to 100 used to score bowel habit. PNE, peripheral nerve evaluation. $\dagger P<0.050$, $\$ P<0.010, \S P<0.001$ (paired comparisons versus baseline; $t$ test); statistical analyses at 12 months are comparisons for 16 patients remaining in the study. 
Table 3 Adverse events among the 36 patients tested

\begin{tabular}{|c|c|}
\hline & $\begin{array}{c}\text { No. of } \\
\text { patients }\end{array}$ \\
\hline Serious adverse events & 9 \\
\hline Wound infection & 2 \\
\hline Electrode wire displacement & 1 \\
\hline Sciatica & 1 \\
\hline Abdominal pain and asthenia & 2 \\
\hline Abdominal pain, anaemia & 1 \\
\hline Sinusitis & 1 \\
\hline Vagal response & 1 \\
\hline Adverse events related or possibly related to the device & 25 \\
\hline Sciatica/pain at IPG site & 5 \\
\hline Wound infection/abcess/hematoma & 3 \\
\hline Abdominal pain & 4 \\
\hline Dysuria & 2 \\
\hline Faecal impaction & 2 \\
\hline Asthenia & 2 \\
\hline Fever & 1 \\
\hline Anal pain & 1 \\
\hline Vaginal infection & 1 \\
\hline Anaemia & 1 \\
\hline Nausea & 1 \\
\hline Faecal incontinence & 1 \\
\hline Deshydratation & 1 \\
\hline
\end{tabular}

IPG, internal pulse generator. 